\title{
QUANTUM ANALOGUES OF SCHUBERT VARIETIES IN THE GRASSMANNIAN
}

\author{
T.H. LENAGAN \\ Maxwell Institute for Mathematical Sciences, School of Mathematics, University of Edinburgh, James Clerk \\ Maxwell Building, King's Buildings, Mayfield Road, Edinburgh EH9 3JZ, Scotland, UK \\ e-mail:tom@maths.ed.ac.uk \\ and L. RIGAL \\ Université Jean-Monnet (Saint-Étienne), Faculté des Sciences et Techniques, Département de \\ Mathématiques, 23 rue du Docteur Paul Michelon, 42023 \\ Saint-Étienne Cédex 2, France \\ e-mail: Laurent.Rigal@univ-st-etienne.fr
}

(Received 27 October, 2006; revised 28 June, 2007; accepted 9 July, 2007)

\begin{abstract}
We study quantum Schubert varieties from the point of view of regularity conditions. More precisely, we show that these rings are domains that are maximal orders and are AS-Cohen-Macaulay and we determine which of them are AS-Gorenstein. One key fact that enables us to prove these results is that quantum Schubert varieties are quantum graded algebras with a straightening law that have a unique minimal element in the defining poset. We prove a general result showing when such quantum graded algebras are maximal orders. Finally, we exploit these results to show that quantum determinantal rings are maximal orders.
\end{abstract}

2000 Mathematics Subject Classification. 16W35; 16P40; 16S38; 17B37; 20 G42.

Introduction. Since the appearance of quantum groups in the eighties, there have been several attempts to define quantum analogues of coordinate rings of grassmannian varieties and, more generally, of flag varieties. Here, we are interested in such deformations for grassmannian varieties and we follow the approach of Lakshmibai and Reshetikhin (see [8]). Hence, we start with the (usual) quantum deformation, denoted by $\mathcal{O}_{q}\left(M_{m, n}(\mathbb{k})\right)$, of the coordinate ring of $m \times n$ matrices. Then, denoting by $G_{m, n}(\mathbb{k})$ the grassmanian of $m$-dimensional subspaces in $\mathbb{k}^{n}$, the quantum deformation of the coordinate ring of $G_{m, n}(\mathbb{k})$ that we consider is the $\mathbb{k}$-subalgebra of $\mathcal{O}_{q}\left(M_{m, n}(\mathbb{k})\right)$ generated by the maximal quantum minors. We denote this algebra by $\mathcal{O}_{q}\left(G_{m, n}(\mathbb{k})\right)$ and call it the quantum grassmannian for simplicity. Precise definitions are recalled in Section 1.

Our main interest, in this paper, is the study of a family of quotients of $\mathcal{O}_{q}\left(G_{m, n}(\mathbb{k})\right)$ that appear in [8] and are natural quantum analogues of coordinate rings of Schubert varieties in $G_{m, n}(\mathbb{k})$. These quantum Schubert varieties have already been studied, to some extent, in [10]. There, they were used as a central tool to show that $\mathcal{O}_{q}\left(G_{m, n}(\mathbb{k})\right)$ is a quantum graded algebra with a straightening law. Details on the notion of quantum graded algebra with a straightening law (quantum graded A.S.L. for short) can be

*This research was supported by the Leverhulme Research Interchange Grant F/00158/X. 
found in Section 2 below and in [10] where this notion was introduced. Roughly speaking, such an algebra is endowed with a standard monomial basis built on the elements of a finite partially ordered subset of generators. In addition, the quantum graded A.S.L. structure provides a good control on the way such standard monomials multiply with each other by means of the so-called straightening law and commutation law.

In fact, the notion of quantum graded A.S.L. is particularly well adapted to studying both $\mathcal{O}_{q}\left(G_{m, n}(\mathbb{k})\right)$ and its associated quantum Schubert varieties, which are also quantum graded A.S.L. as shown in [10]. Here, we use this notion to study quantum Schubert varieties from the point of view of noncommutative algebraic geometry. Namely, we first show that they are integral domains that are maximal orders in their division ring of fractions. Recall that the notion of maximal order generalises, in the noncommutative setting, that of a normal domain in commutative algebra. Next, we study their regularity properties in the sense of Artin and Schelter (namely, homological properties such as the Cohen-Macaulay and the Gorenstein properties).

Also, it turns out that quantum Schubert varieties are strongly linked to another family of interesting quantum algebras: the quantum determinantal rings. These are quotients of $\mathcal{O}_{q}\left(M_{m, n}(\mathbb{k})\right)$ by the ideal generated by quantum minors of a given size. Hence, as a consequence of our results, we are able to show that quantum determinantal rings are maximal orders in their division ring of fractions. This generalises results obtained in [9] where it was shown that quantum determinantal rings are maximal orders under the restrictive hypotheses that $\mathbb{k}$ is the field of complex numbers and the deformation parameter $q$ is transcendental over $\mathbb{Q}$.

The paper is organised as follows. Section 1 is mainly devoted to recalling basic definitions and crucial results concerning the quantum algebras we intend to study. Section 2 starts with a short reminder about the notion of quantum graded A.S.L. Here, we establish a general criterion that allows us to prove that, in certain circumstances, a quantum graded algebra with a straightening law that is a domain and whose underlying poset has a single minimal element is a maximal order in its division ring of fractions. In Section 3 we use the results of Section 2 to show that quantum Schubert varieties are integral domains and are maximal orders in their division ring of fractions. Also, we investigate their regularity properties. It was shown in [10] that quantum Schubert varieties are AS-Cohen-Macaulay. Here we determine which of them are AS-Gorenstein. Section 4 is devoted to proving that quantum determinantal rings are maximal orders in their division ring of fractions. This is shown by using the material of the two preceding sections.

Recall from [11, Chapter 5, Section 1] that a commutative noetherian domain $A$ is a maximal order in its quotient field if and only if it is integrally closed. For this reason, if $A$ is a (noncommutative) domain, then we will say that $A$ is normal if it is a maximal order in its division ring of fractions. This convention differs slightly from classical uses in noncommutative algebra.

Throughout $\mathbb{k}$ denotes a field. The cardinality of a finite set $X$ is denoted by $|X|$.

1. Basic definitions. In this section, we collect some basic definitions and properties about the objects we intend to study. Most proofs will be omitted since these results already appear in [5] and [10]. Appropriate references will be given in the text.

Let $m, n$ be positive integers. The standard quantization of the coordinate ring of the affine variety $M_{m, n}(\mathbb{k})$ of $m \times n$ matrices with entries in $\mathbb{k}$ is denoted $\mathcal{O}_{q}\left(M_{m, n}(\mathbb{k})\right)$. 
It is the $\mathbb{k}$-algebra generated by $m n$ indeterminates $X_{i j}$, with $1 \leq i \leq m$ and $1 \leq j \leq n$, subject to the relations:

$$
\begin{array}{ll}
X_{i j} X_{i l}=q X_{i l} X_{i j}, & \text { for } 1 \leq i \leq m, \text { and } 1 \leq j<l \leq n \\
X_{i j} X_{k j}=q X_{k j} X_{i j}, & \text { for } 1 \leq i<k \leq m, \text { and } 1 \leq j \leq n \\
X_{i j} X_{k l}=X_{k l} X_{i j}, & \text { for } 1 \leq k<i \leq m, \text { and } 1 \leq j<l \leq n \\
X_{i j} X_{k l}-X_{k l} X_{i j}=\left(q-q^{-1}\right) X_{i l} X_{k j}, & \text { for } 1 \leq i<k \leq m, \text { and } 1 \leq j<l \leq n .
\end{array}
$$

To simplify, we write $M_{n}(\mathbb{k})$ for $M_{n, n}(\mathbb{k})$. The $m \times n$ matrix $\mathbf{X}=\left(X_{i j}\right)$ is called the generic matrix associated with $\mathcal{O}_{q}\left(M_{m, n}(\mathbb{k})\right)$.

As is well known, there exists a $\mathbb{k}$-algebra transpose isomorphism between $\mathcal{O}_{q}\left(M_{m, n}(\mathbb{k})\right)$ and $\mathcal{O}_{q}\left(M_{n, m}(\mathbb{k})\right)$, see [10, Remark 3.1.3]. Hence, from now on, we assume that $m \leq n$, without loss of generality.

An index pair (in $\{1, \ldots, m\} \times\{1, \ldots, n\}$ ) is a pair $(I, J)$ such that $I \subseteq\{1, \ldots, m\}$ and $J \subseteq\{1, \ldots, n\}$ are subsets with the same cardinality. Hence, an index pair is given by an integer $t$ such that $1 \leq t \leq m$ and ordered sets $I=\left\{i_{1}<\ldots<i_{t}\right\} \subseteq\{1, \ldots, m\}$ and $J=\left\{j_{1}<\ldots<j_{t}\right\} \subseteq\{1, \ldots, n\}$. To any such index pair we associate the quantum minor

$$
[I \mid J]=\sum_{\sigma \in \mathfrak{S}_{t}}(-q)^{\ell(\sigma)} X_{i_{\sigma(1)} j_{1}} \ldots X_{i_{\sigma(t)} j_{t}}
$$

The set of all index pairs is denoted by $\Delta_{m, n}$. Since $\Delta_{m, n}$ is in one-to-one correspondence with the set of all quantum minors of $\mathcal{O}_{q}\left(M_{m, n}(\mathbb{k})\right)$, we will often identify these two sets. The set $\Delta_{m, n}$ is equipped with the partial order $\leq_{\text {st }}$ defined in [10, Section 3.5]. Namely, if $(I, J)$ and $(K, L)$ are index pairs with $I=\left\{i_{1}<\ldots<i_{u}\right\}, J=\left\{j_{1}<\ldots<\right.$ $\left.j_{u}\right\}, K=\left\{k_{1}<\ldots<k_{v}\right\}$ and $L=\left\{l_{1}<\ldots<l_{v}\right\}$ then

$$
(I, J) \leq_{\mathrm{st}}(K, L) \Longleftrightarrow\left\{\begin{array}{l}
u \geq v, \\
i_{s} \leq k_{s} \text { for } 1 \leq s \leq v, \\
j_{s} \leq l_{s} \text { for } 1 \leq s \leq v .
\end{array}\right.
$$

We now consider the quantization of the coordinate ring of the grassmannian of $m$-dimensional subspaces of $\mathbb{k}^{n}$, denoted by $\mathcal{O}_{q}\left(G_{m, n}(\mathbb{k})\right)$. This is defined to be the subalgebra of $\mathcal{O}_{q}\left(M_{m, n}(\mathbb{k})\right)$ generated by the $m \times m$ quantum minors.

An index set (in $\{1, \ldots, n\}$ ) is a subset $I=\left\{i_{1}<\ldots<i_{m}\right\} \subseteq\{1, \ldots, n\}$. To any index set we associate the maximal quantum minor $[\{1, \ldots, m\} \mid I]$ of $\mathcal{O}_{q}\left(M_{m, n}(\mathbb{k})\right)$ that is, thus, an element of $\mathcal{O}_{q}\left(G_{m, n}(\mathbb{k})\right)$. The set of all index sets is denoted by $\Pi_{m, n}$. Since $\Pi_{m, n}$ is in one-to-one correspondence with the set of all maximal quantum minors of $\mathcal{O}_{q}\left(M_{m, n}(\mathbb{k})\right)$, we will often identify these two sets. The map $\Pi_{m, n} \longrightarrow \Delta_{m, n}$ given by $I \mapsto(\{1, \ldots, m\}, I)$, identifies $\Pi_{m, n}$ with a subset of $\Delta_{m, n}$. Hence, the partial order $\leq_{\text {st }}$ induces a partial order on $\Pi_{m, n}$ that we still denote by $\leq_{\text {st }}$. Clearly, if $I=\left\{i_{1}<\ldots<i_{m}\right\}$ and $J=\left\{j_{1}<\ldots<j_{m}\right\}$ are two index sets, we have

$$
I \leq_{\mathrm{st}} J \Longleftrightarrow i_{s} \leq j_{s} \text { for } \quad 1 \leq s \leq m .
$$

The order in which the $\mathbb{k}$-algebras $\mathcal{O}_{q}\left(M_{m, n}(\mathbb{k})\right)$ and $\mathcal{O}_{q}\left(G_{m, n}(\mathbb{k})\right)$ have been introduced above is forced upon us by the definition of the quantum grassmannian. Despite this, from our point of view, the more fundamental object is $\mathcal{O}_{q}\left(G_{m, n}(\mathbb{k})\right)$, rather than $\mathcal{O}_{q}\left(M_{m, n}(\mathbb{k})\right)$ and we concentrate on $\mathcal{O}_{q}\left(G_{m, n}(\mathbb{k})\right)$ in this paper. Indeed, many desirable properties shared by these two algebras are more easily proven for 
$\mathcal{O}_{q}\left(G_{m, n}(\mathbb{k})\right)$. Then, the corresponding property can be transfered to $\mathcal{O}_{q}\left(M_{m, n}(\mathbb{k})\right)$ using the dehomogenisation map $D_{m, n}$, introduced in [5], that relates the two algebras. We briefly recall the definition of this map. Recall from [10, Section 3.5] that to any $(I, J) \in \Delta_{m, n}$ we associate $K_{(I, J)} \in \Pi_{m, m+n}$ where, if $I=\left\{i_{1}<\ldots<i_{t}\right\}$ and $J=\left\{j_{1}<\ldots<j_{t}\right\}$, for $1 \leq t \leq m$, we set $K_{(I, J)}:=\left\{j_{1}, \ldots, j_{t}, n+1 \ldots, n+m\right\} \backslash\{n+$ $\left.m+1-i_{1}, \ldots, n+m+1-i_{t}\right\}$. Then, letting $M=\{n+1, \ldots, n+m\}$, the map $\delta_{m, n}$ : $\Delta_{m, n} \longrightarrow \Pi_{m, m+n} \backslash\{M\},(I, J) \mapsto K_{(I, J)}$ is an isomorphism of partially ordered sets, see $[10,3.5 .2]$. Let $\phi$ be the automorphism of $\mathcal{O}_{q}\left(M_{m, n}(\mathbb{k})\right)$ defined by $\phi\left(X_{i j}\right)=q^{-1} X_{i j}$ for $1 \leq i \leq m$ and $1 \leq j \leq n$. It can be shown that there exists a $\mathbb{k}$-algebra isomorphism

$$
D_{m, n}: \mathcal{O}_{q}\left(M_{m, n}(\mathbb{k})\right)\left[y, y^{-1} ; \phi\right] \longrightarrow \mathcal{O}_{q}\left(G_{m, m+n}(\mathbb{k})\right)\left[[M]^{-1}\right]
$$

given by $[I \mid J] \mapsto\left[K_{(I, J)}\right][M]^{-1}$ and $y \mapsto[M]$. For details about this map see $[\mathbf{1 0}$, Section 3.5].

One crucial property in the study of $\mathcal{O}_{q}\left(G_{m, n}(\mathbb{k})\right)$ and $\mathcal{O}_{q}\left(M_{m, n}(\mathbb{k})\right)$ is the existence of standard monomial bases. By a standard monomial in $\mathcal{O}_{q}\left(G_{m, n}(\mathbb{k})\right)$ we mean either 1 or a product of the form $\left[I_{1}\right] \ldots\left[I_{\ell}\right]$, where $\ell \in \mathbb{N}^{*}$ and $I_{1} \leq_{\mathrm{st}} \ldots \leq_{\mathrm{st}} I_{\ell} \in \Pi_{m, n}$. By $[\mathbf{1 0}, 3.2 .4]$, the set of standard monomials is a $\mathbb{k}$-basis of $\mathcal{O}_{q}\left(G_{m, n}(\mathbb{k})\right)$, called the standard monomial basis of $\mathcal{O}_{q}\left(G_{m, n}(\mathbb{k})\right)$. A similar notion of standard monomial can be introduced in $\mathcal{O}_{q}\left(M_{m, n}(\mathbb{k})\right)$ using $\Delta_{m, n}$.

Here again, it turns out that the set of standard monomials is a $\mathbb{k}$-basis of $\mathcal{O}_{q}\left(M_{m, n}(\mathbb{k})\right)$, called the standard monomial basis of $\mathcal{O}_{q}\left(M_{m, n}(\mathbb{k})\right)$. This fact can easily be proved using the standard monomial basis of the quantum grassmannian and the map $D_{m, n}$, see the proof of $[\mathbf{1 0}, 3.5 .3]$ for part of the argument. However, note that it is also a special case of $[\mathbf{4}$, Theorem 9$]$.

We now introduce the main object of investigation of the present work, namely, quantum analogues of coordinate rings of Schubert varieties in the grassmannian.

Definition 1.1. Let $\gamma \in \Pi_{m, n}$ and put $\Pi_{m, n}^{\gamma}=\left\{\alpha \in \Pi_{m, n} \mid \alpha \geq_{\text {st }} \gamma\right\}$. The quantum Schubert variety associated to $\gamma$ is

$$
\mathcal{O}_{q}\left(G_{m, n}(\mathbb{k})\right)_{\gamma}:=\mathcal{O}_{q}\left(G_{m, n}(\mathbb{k})\right) /\left\langle\Pi_{m, n}^{\gamma}\right\rangle .
$$

REMARK 1.2.

(i) Quantum Schubert varieties, as defined above, appear in [8, p.162]. Notice, however, that our conventions differ slightly from those of [8]. However, it is easy to see that the two different conventions produce isomorphic algebras.

(ii) Definition 1.1 is inspired by the classical description of the coordinate rings of Schubert varieties in the grassmannian. For details about this matter, see [3, Section 6.3.4].

It turns out that quantum Schubert varieties also have standard monomial bases. In fact these bases are inherited from the corresponding bases for $\mathcal{O}_{q}\left(G_{m, n}(\mathbb{k})\right)$. One convenient way to show this is by means of the notion of quantum graded algebra with a straightening law. Hence, we postpone the details about this point until Section 2 where this notion is discussed.

We end this section by showing a technical result that we will use later on. It is a quantum analogue of Muir's Law of Extensible Minors. In fact, the result we prove, Proposition 1.3 below, is only a special case of the Quantum Muir's Law of Extensible Minors. For a general result, the reader is referred to [7, Theorem 3.4]. Even though the result we prove can be deduced from [7, Theorem 3.4], we have inserted a proof for the convenience of the reader, since our proof is relatively short. 
Recall that $n$ is a positive integer and put $F=\{1, \ldots, n\}$. As is well known, the quantum determinant $[F \mid F]$ is a central element of $\mathcal{O}_{q}\left(M_{n}(\mathbb{k})\right)$, see, for example, $[\mathbf{1 3}$, 4.6.1]. Hence, we may form the localisation $\mathcal{O}_{q}\left(G L_{n}(\mathbb{k})\right):=\mathcal{O}_{q}\left(M_{n}(\mathbb{k})\right)\left[[F \mid F]^{-1}\right]$. By $[\mathbf{1 3}, 5.3 .2], \mathcal{O}_{q}\left(G L_{n}(\mathbb{k})\right)$ is a Hopf algebra whose antipode is the anti-automorphism $S$ induced by

$$
S\left(X_{i j}\right)=(-q)^{i-j}[F \backslash\{j\} \mid F \backslash\{i\}][F \mid F]^{-1} .
$$

In addition, if $I=\left\{i_{1}, \ldots, i_{t}\right\}$ and $J=\left\{j_{1}, \ldots, j_{t}\right\}$ are subsets of $F$ then

$$
S([I \mid J])=(-q)^{\left(i_{1}+\ldots+i_{t}\right)-\left(j_{1}+\ldots+j_{t}\right)}[F \backslash J \mid F \backslash I][F \mid F]^{-1},
$$

see, for example, [5, Lemma 4.1].

Now, for $1 \leq \ell \leq n$, let $\varepsilon_{\ell}$ denote the element of $\mathbb{N}^{n}$ whose only nonzero coordinate equals one and is in the $\ell$-th position. There is a natural $\mathbb{N}^{n} \times \mathbb{N}^{n}$-grading on $\mathcal{O}_{q}\left(M_{n}\right)$ relative to which, for $1 \leq i, j \leq n$, the degree of $X_{i j}$ is $\left(\varepsilon_{i}, \varepsilon_{j}\right)$. Clearly, if $I, J$ are subsets of $F$ of the same cardinality, the degree of $[I \mid J]$ is $\left(\sum_{i \in I} \varepsilon_{i}, \sum_{j \in J} \varepsilon_{j}\right)$.

Proposition 1.3. Let $P, Q$ be two subsets of $F$ of the same cardinality and denote by $\bar{P}, \bar{Q}$ their respective complements in $F$. Consider $d \in \mathbb{N}^{*}$ and, for $1 \leq s \leq d$, elements $c_{s} \in \mathbb{k}$ and subsets $I_{s}, K_{s} \subseteq P$ and $J_{s}, L_{s} \subseteq Q$ such that $\left|I_{s}\right|=\left|J_{s}\right|$ and $\left|\mathrm{K}_{s}\right|=\left|L_{s}\right|$. If the relation $\sum_{s=1}^{d} c_{s}\left[I_{s} \mid J_{s}\right]\left[K_{s} \mid L_{s}\right]=0$ holds in $\mathcal{O}_{q}\left(M_{n}(\mathbb{k})\right)$, then the relation

$$
\sum_{s=1}^{d} c_{s}\left[I_{s} \cup \bar{P} \mid J_{s} \cup \bar{Q}\right]\left[K_{s} \cup \bar{P} \mid L_{s} \cup \bar{Q}\right]=0
$$

holds in $\mathcal{O}_{q}\left(M_{n}(\mathbb{k})\right)$.

Proof. We may suppose, without loss of generality, that the products $\left[I_{s} \mid J_{s}\right]\left[K_{s} \mid L_{s}\right]$ in the relation $\sum_{s=1}^{d} c_{s}\left[I_{s} \mid J_{s}\right]\left[K_{s} \mid L_{s}\right]=0$ have the same $\mathbb{N}^{n} \times \mathbb{N}^{n}$-degree. Now, let $p$ be the common cardinality of $P$ and $Q$. The subalgebra of $\mathcal{O}_{q}\left(M_{n}(\mathbb{k})\right)$ generated by those $X_{i j}$ such that $i \in P$ and $j \in Q$ is isomorphic to $\mathcal{O}_{q}\left(M_{p}(\mathbb{k})\right)$. Hence, we may consider the relation $\sum_{s=1}^{d} c_{s}\left[I_{s} \mid J_{s}\right]\left[K_{s} \mid L_{s}\right]=0$ as a relation in $\mathcal{O}_{q}\left(M_{p}(\mathbb{k})\right)$ and apply to this relation the antipode of $\mathcal{O}_{q}\left(G L_{p}(\mathbb{k})\right)$. This yields the relation

$$
\sum_{s=1}^{d} c_{s}\left[Q \backslash L_{S} \mid P \backslash K_{s}\right]\left[Q \backslash J_{s} \mid P \backslash I_{s}\right]=0
$$

in $\mathcal{O}_{q}\left(M_{n}(\mathbb{k})\right)$. (Notice that the $\mathbb{N}^{n} \times \mathbb{N}^{n}$-homogeniety of the relation has been used here to cancel out the various powers of $q$ occuring from the application of the antipode.) Now, applying the antipode of $\mathcal{O}_{q}\left(G L_{n}(\mathbb{k})\right)$ to this relation gives us the relation

$$
\sum_{s=1}^{d} c_{s}\left[I_{s} \cup \bar{P} \mid J_{s} \cup \bar{Q}\right]\left[K_{s} \cup \bar{P} \mid L_{s} \cup \bar{Q}\right]=0
$$

in $\mathcal{O}_{q}\left(M_{n}(\mathbb{k})\right)$.

2. Quantum graded algebras with a straightening law. In this section, we start reviewing the notion of quantum graded algebra with a straightening law, as introduced and studied in [10]. Next, we give a criterion that allows us to show that, under certain 
hypotheses, a quantum graded algebra with a straightening law that is a domain and whose underlying partially ordered set has a single minimal element is a maximal order in its division ring of fractions.

2.1. Short reminder. In this subsection, we recall the notion of a quantum graded algebra with a straightening law (on a partially ordered set $\Pi$ ). We also recall various properties of such algebras that we will use later.

Let $A$ be an algebra and $\Pi$ a finite subset of elements of $A$ with a partial order $<_{\text {st }}$. A standard monomial on $\Pi$ is an element of $A$ that is either 1 or of the form $\alpha_{1} \ldots \alpha_{s}$, for some $s \geq 1$, where $\alpha_{1}, \ldots, \alpha_{s} \in \Pi$ and $\alpha_{1} \leq_{\text {st }} \ldots \leq_{\text {st }} \alpha_{s}$.

Definition 2.1.1. Let $A$ be an $\mathbb{N}$-graded $\mathbb{k}$-algebra and $\Pi$ a finite subset of $A$ equipped with a partial order $<_{\mathrm{st}}$. We say that $A$ is a quantum graded algebra with a straightening law (quantum graded A.S.L. for short) on the poset $\left(\Pi,<_{\mathrm{st}}\right)$ if the following conditions are satisfied.

(1) The elements of $\Pi$ are homogeneous with positive degree.

(2) The elements of $\Pi$ generate $A$ as a $\mathbb{k}$-algebra.

(3) The set of standard monomials on $\Pi$ is a linearly independent set.

(4) If $\alpha, \beta \in \Pi$ are not comparable for $<_{\text {st }}$, then $\alpha \beta$ is a linear combination of terms $\lambda$ or $\lambda \mu$, where $\lambda, \mu \in \Pi, \lambda \leq_{\text {st }} \mu$ and $\lambda<_{\text {st }} \alpha, \beta$.

(5) For all $\alpha, \beta \in \Pi$, there exists $c_{\alpha \beta} \in \mathbb{k}^{*}$ such that $\alpha \beta-c_{\alpha \beta} \beta \alpha$ is a linear combination of terms $\lambda$ or $\lambda \mu$, where $\lambda, \mu \in \Pi, \lambda \leq_{\text {st }} \mu$ and $\lambda<_{\text {st }} \alpha, \beta$.

By [10, Proposition 1.1.4], if $A$ is a quantum graded A.S.L. on the partially ordered set $\left(\Pi,<_{\text {st }}\right)$, then the set of standard monomials on $\Pi$ forms a $\mathbb{k}$-basis of $A$. Hence, in the presence of a standard monomial basis, the structure of a quantum graded A.S.L. may be seen as providing substantial further information on the way standard monomials multiply and commute.

EXAMPLE 2.1.2. As is well known, the algebra $\mathcal{O}_{q}\left(M_{m, n}(\mathbb{k})\right)$ is $\mathbb{N}$-graded, by putting the canonical generators in degree one. Now, since $\mathcal{O}_{q}\left(G_{m, n}(\mathbb{k})\right)$ is a subalgebra of $\mathcal{O}_{q}\left(M_{m, n}(\mathbb{k})\right)$ generated by homogeneous elements, $\mathcal{O}_{q}\left(G_{m, n}(\mathbb{k})\right)$ inherits a natural $\mathbb{N}$ grading from that of $\mathcal{O}_{q}\left(M_{m, n}(\mathbb{k})\right)$. In fact, beyond the existence of standard monomial bases for $\mathcal{O}_{q}\left(G_{m, n}(\mathbb{k})\right)$ and $\mathcal{O}_{q}\left(M_{m, n}(\mathbb{k})\right)$, as mentioned in Section 1, we have that $\mathcal{O}_{q}\left(G_{m, n}(\mathbb{k})\right)$ is a quantum graded A.S.L. on $\left(\Pi_{m, n}, \leq_{\mathrm{st}}\right)$ and that $\mathcal{O}_{q}\left(M_{m, n}(\mathbb{k})\right)$ is a quantum graded A.S.L. on $\left(\Delta_{m, n}, \leq_{\text {st }}\right)$, see [10, Theorem 3.4.4 and 3.5.3].

From our point of view, one important feature of quantum graded A.S.L. is the following. Let $A$ be a $\mathbb{k}$-algebra that is a quantum graded A.S.L. on the set $\left(\Pi, \leq_{\text {st }}\right)$. A subset $\Omega$ of $\Pi$ will be called a $\Pi$-ideal if it is an ideal of the partially ordered set $\left(\Pi, \leq_{\mathrm{st}}\right)$ in the sense of lattice theory; that is, if it satisfies the following property: if $\alpha \in \Omega$ and if $\beta \in \Pi$, with $\beta \leq_{\text {st }} \alpha$, then $\beta \in \Omega$. We can consider the quotient $A /\langle\Omega\rangle$ of $A$ by the ideal generated by $\Omega$. Clearly, it is still a graded algebra and it is generated by the images in $A /\langle\Omega\rangle$ of the elements of $\Pi \backslash \Omega$. The important point here is that $A /\langle\Omega\rangle$ inherits from $A$ a natural quantum graded A.S.L. structure on $\Pi \backslash \Omega$ (or, more precisely, on the canonical image of $\Pi \backslash \Omega$ in $A /\langle\Omega\rangle$ ). In particular, the set of homomorphic images in $A /\langle\Omega\rangle$ of the standard monomials of $A$ that either equal 1 or are of the form $\alpha_{1} \ldots \alpha_{t}$ $\left(t \in \mathbb{N}^{*}\right)$ and $\alpha_{1} \notin \Omega$ form a $\mathbb{k}$-basis for $A /\langle\Omega\rangle$. The reader will find all the necessary details in [10, Section 1.2]. 
EXAMPLE 2.1.3. Let $\gamma \in \Pi_{m, n}$. It is clear that the set $\Pi_{m, n}^{\gamma}$ introduced in Definition 1.1 is a $\Pi_{m, n}$-ideal. Hence, the discussion above shows that the quantum Schubert variety $\mathcal{O}_{q}\left(G_{m, n}(\mathbb{k})\right)_{\gamma}$ is a quantum graded A.S.L. on the canonical image in $\mathcal{O}_{q}\left(G_{m, n}(\mathbb{k})\right)_{\gamma}$ of $\Pi_{m, n} \backslash \Pi_{m, n}^{\gamma}$. In particular, the canonical images in $\mathcal{O}_{q}\left(G_{m, n}(\mathbb{k})\right)_{\gamma}$ of the standard monomials of $\mathcal{O}_{q}\left(G_{m, n}(\mathbb{k})\right)$ that either equal to 1 or are of the form $\left[I_{1}\right] \ldots\left[I_{t}\right]$, for some $t \geq 1$ and with $\gamma \leq_{\text {st }}\left[I_{1}\right]$, form a $\mathbb{k}$-basis of $\mathcal{O}_{q}\left(G_{m, n}(\mathbb{k})\right)_{\gamma}$.

REMARK 2.1.4. Let $\gamma \in \Pi_{m, n}$. As mentioned in Example 2.1.3, the quantum Schubert variety $\mathcal{O}_{q}\left(G_{m, n}(\mathbb{k})\right)_{\gamma}$ is a quantum graded A.S.L. on the canonical image in $\mathcal{O}_{q}\left(G_{m, n}(\mathbb{k})\right)_{\gamma}$ of $\Pi_{m, n} \backslash \Pi_{m, n}^{\gamma}$. At this point, it is worth noting that the set $\Pi_{m, n} \backslash \Pi_{m, n}^{\gamma}$ has a single minimal element, namely $\gamma$.

We end this subsection by recalling from [10, Proposition 1.1.5] the formula that gives the Gelfand-Kirillov dimension of a quantum graded A.S.L. Note that, if $\left(\Pi, \leq_{\mathrm{st}}\right)$ is a partially ordered set, the rank of an element $\pi \in \Pi$, denoted $\mathrm{rk} \pi$, is the greatest integer $k \in \mathbb{N}$ such that there exists a chain $\pi_{1}<_{\text {st }} \ldots<_{\text {st }} \pi_{k-1}<_{\text {st }} \pi_{k}=\pi$ of elements of $\Pi$. Then, we define the rank of $\Pi$ by $\operatorname{rk} \Pi=\max \{\operatorname{rk} \pi, \pi \in \Pi\}$. Then, we have the following proposition.

Proposition 2.1.5. Let $A$ be a quantum graded A.S.L. on $\left(\Pi, \leq_{\mathrm{st}}\right)$; then $\mathrm{GK} \operatorname{dim} A=$ rkП.

COROLlaRY 2.1.6. Let $\gamma=\left\{\gamma_{1}, \ldots, \gamma_{m}\right\} \in \Pi_{m, n}$. Then

$$
\mathrm{GKdim}_{q}\left(G_{m, n}(\mathbb{k})\right)_{\gamma}=m(n-m)+\frac{m(m+1)}{2}-\left(\sum_{i=1}^{m} \gamma_{i}\right)+1 .
$$

Proof. It is well known that $\operatorname{rk}\left(\Pi_{m, n} \backslash \Pi_{m, n}^{\gamma}\right)=m(n-m)+\frac{m(m+1)}{2}-\left(\sum_{i=1}^{m} \gamma_{i}\right)+$ 1 , see $[1,5.12]$; so, the result follows from Proposition 2.1.5.

2.2. Quantum graded A.S.L. and the maximal order property. In this subsection we are interested in quantum graded A.S.L. whose associated poset has a single minimal element. Hence, let $A$ be a quantum graded A.S.L. on the poset $\left(\Pi, \leq_{\text {st }}\right)$, and assume that $\left(\Pi, \leq_{\text {st }}\right)$ has a single minimal element, denoted $\gamma$. We know that $\gamma$ is a regular normal element of $A$, by [10, Lemma 1.2.1]. Hence, we may form the localisation, $A\left[\gamma^{-1}\right]$, of $A$ with respect to the powers of $\gamma$ and the canonical map $A \longrightarrow A\left[\gamma^{-1}\right]$ is injective. Notice that quantum Schubert varieties are examples of such algebras, as mentioned in Remark 2.1.4.

Our first interest is in studying the ideal $\langle\gamma\rangle$ of $A$.

To each element $\sigma \in \Pi$, we associate the subset $\Pi^{\sigma}$ of $\Pi$ defined by

$$
\Pi^{\sigma}=\left\{\pi \in \Pi \mid \pi \Varangle_{\mathrm{st}} \sigma\right\} .
$$

It is clear that $\Pi^{\sigma}$ is a $\Pi$-ideal. In addition, we let $I_{\sigma}$ be the ideal of $A$ generated by $\Pi^{\sigma}$ :

$$
I_{\sigma}=\left\langle\Pi^{\sigma}\right\rangle .
$$

It is clear that, if $\sigma$ and $\tau$ are elements of $\Pi$ such that $\sigma \leq_{\text {st }} \tau$, then $\Pi^{\sigma} \subseteq \Pi^{\tau}$ and hence $I_{\sigma} \subseteq I_{\tau}$. Finally, a last piece of notation: let $\sigma \in \Pi$; an element $\tau$ is called an upper neighbour of $\sigma$ if $\sigma<_{\text {st }} \tau$ and there is no element $v \in \Pi$ such that $\sigma<_{\text {st }} v<_{\text {st }} \tau$. 
Clearly, for any $\sigma \in \Pi$, the set of upper neighbours of $\sigma$ is a (finite) subset of $\Pi$ that is empty if and only if $\sigma$ is maximal.

LEMMA 2.2.1. We keep the notation introduced above.

(i) Let $\psi$ be the automorphism of $A$ associated to the regular normal element $\gamma$; that $i s, \gamma a=\psi(a) \gamma$, for all $a \in A$. Then $\psi\left(I_{\tau}\right)=I_{\tau}$ for all $\tau \in \Pi$.

(ii) Suppose that $\{\gamma\} \varsubsetneqq \Pi$. Then the following formula holds, where the intersection is taken over all the upper neighbours $\tau$ of $\gamma$ :

$$
\langle\gamma\rangle=\bigcap I_{\tau} .
$$

Proof. (i) By condition (5) of Definition 2.1.1, the element $\gamma$ commutes up to a non zero scalar with each element of $\Pi$. Thus, each $\tau \in \Pi$ is an eigenvector (with non zero eigenvalue) of $\psi$; so, the statement is clear.

(ii) There is at least one upper neighbour of $\gamma$, since $\{\gamma\} \varsubsetneqq \Pi$. As $\gamma \in \Pi^{\tau}$, for all upper neighbours $\tau$ of $\gamma$, the inclusion $\langle\gamma\rangle \subseteq \bigcap I_{\tau}$ is clear. Let us now obtain the reverse inclusion. First, notice that any element of $\Pi$ different from $\gamma$ must be greater than or equal to some upper neighbour of $\gamma$; this is an easy consequence of the fact that $\Pi$ is a finite partially ordered set whose unique minimal element is $\gamma$. On the other hand, for all $\tau \in \Pi$, since $\Pi^{\tau}$ is a $\Pi$-ideal, the ideal $I_{\tau}$ is the vector space generated by standard monomials involving an element of $\Pi^{\tau}$, see [10, Proposition 1.2.5]. Hence, $I_{\tau}$ is the vector space generated by standard monomials of the form $\alpha_{1} \ldots \alpha_{r}$, with $\alpha_{1} \leq_{\text {st }} \ldots \leq_{\text {st }} \alpha_{r} \in \Pi$ and such that $\alpha_{1} \Varangle_{\text {st }} \tau$. Since, in addition, the standard monomials form a basis of $A$, it follows that $\bigcap I_{\tau}$ (where the intersection is taken over all the upper neighbours of $\gamma$ ) is the vector space generated by standard monomials of the form $\alpha_{1} \ldots \alpha_{r}$, with $\alpha_{1} \leq_{\text {st }} \ldots \leq_{\text {st }} \alpha_{r} \in \Pi$ and such that $\alpha_{1}$ is not greater than or equal to any upper neighbour of $\gamma$. By the above comment, this forces $\alpha_{1}=\gamma$. The inclusion $\bigcap I_{\tau} \subseteq\langle\gamma\rangle$ now follows.

Proposition 2.2.2. We keep the notation introduced above. Assume that $A$ is a domain such that $I_{\tau}$ is a completely prime ideal of $A$ for any upper neighbour $\tau$ of $\gamma$, and that $A\left[\gamma^{-1}\right]$ is a maximal order in its division ring of fractions. Then $A$ is a maximal order in its division ring of fractions.

Proof. First, recall from [10, Lemma 1.2.3] that $A$ is noetherian. Notice, in addition, that if $\Pi=\{\gamma\}$, then $A$ is a commutative polynomial ring in one indeterminate; so that $A$ is clearly a maximal order in its division ring of fractions. Now, assume that $\{\gamma\} \varsubsetneqq \Pi$. By Lemma 2.2.1 and the hypotheses made on $A$, we are in position to apply $[\mathbf{1 4}$, Lemma 1.1] that gives the result.

3. Quantum Schubert varieties in the grassmannian. Let $m, n$ be positive integers. As discussed above, to each $\gamma \in \Pi_{m, n}$, we may associate the $\mathbb{k}$-algebra $\mathcal{O}_{q}\left(G_{m, n}(\mathbb{k})\right)_{\gamma}$, which is a quantum deformation of the coordinate ring of a Schubert variety. The aim of this section is to study these rings. In the first subsection, we shall will show that they are normal integral domains. In the second section, we shall will study them from the point of view of regularity conditions.

3.1. Integrality and normality. Our aim in this subsection is to prove that the quantum Schubert variety $\mathcal{O}_{q}\left(G_{m, n}(\mathbb{k})\right)_{\gamma}$ is a normal domain for any $\gamma \in \Pi_{m, n}$. Here, by normal domain, we mean an integral domain that is a maximal order in its division 
ring of fractions. To achieve this goal, we will be naturally led to use certain subalgebras of $\mathcal{O}_{q}\left(M_{m, n}(\mathbb{k})\right)$ that we now define.

Let us start by introducing some convenient notation. To each $\gamma=\left\{\gamma_{1}, \ldots, \gamma_{m}\right\} \in$ $\Pi_{m, n}$, with $1 \leq \gamma_{1}<\ldots<\gamma_{m} \leq n$, we associate the subset $\mathcal{L}_{\gamma}$ of $\{1, \ldots, m\} \times\{1, \ldots, n\}$ defined by

$$
\mathcal{L}_{\gamma}=\left\{(i, j) \in\{1, \ldots, m\} \times\{1, \ldots, n\} \mid j>\gamma_{m+1-i} \text { and } j \neq \gamma_{\ell} \quad \text { for } \quad 1 \leq \ell \leq m\right\},
$$

that we call the ladder associated with $\gamma$.

It follows from the definition of $\mathcal{L}_{\gamma}$ that for each $(i, j) \in \mathcal{L}_{\gamma}$ the set $\left\{\gamma_{1}, \ldots, \gamma_{m}\right\} \backslash$ $\left\{\gamma_{m+1-i}\right\} \cup\{j\}$ is a subset of $\{1, \ldots, n\}$ containing $m$ distinct elements. Hence, it makes sense to associate to this subset the maximal quantum minor $m_{i j}:=\left[\left\{\gamma_{1}, \ldots, \gamma_{m}\right\} \backslash\right.$ $\left.\left\{\gamma_{m+1-i}\right\} \cup\{j\}\right]$ of $\mathcal{O}_{q}\left(M_{m, n}(\mathbb{k})\right)$. We then set

$$
\mathcal{M}_{\gamma}=\left\{m_{i j} \in \Pi_{m, n} \mid(i, j) \in \mathcal{L}_{\gamma}\right\} \subseteq \Pi_{m, n} .
$$

REMARK 3.1.1. Let $\gamma=\left\{\gamma_{1}, \ldots, \gamma_{m}\right\} \in \Pi_{m, n}$.

(i) Consider $j, l \in\{1, \ldots, m\}$ and suppose $j \notin\left\{\gamma_{1}, \ldots, \gamma_{m}\right\}$. Then, clearly, $j<$ $\gamma_{l}$ implies that $\left[\left\{\gamma_{1}, \ldots, \gamma_{m}\right\} \backslash\left\{\gamma_{l}\right\} \cup\{j\}\right]<_{\text {st }} \gamma$ while $j>\gamma_{l}$ implies that $\left[\left\{\gamma_{1}, \ldots, \gamma_{m}\right\} \backslash\left\{\gamma_{l}\right\} \cup\{j\}\right]>_{\text {st }} \gamma$.

(ii) Hence, the elements of $\mathcal{M}_{\gamma}$ are nothing but the elements of $\Pi_{m, n}$ that are greater than $\gamma$ with respect to the partial order $\leq_{\text {st }}$ and differ from $\gamma$ by exactly one column index.

Definition 3.1.2. Let $\gamma=\left\{\gamma_{1}, \ldots, \gamma_{m}\right\} \in \Pi_{m, n}$, with $1 \leq \gamma_{1}<\ldots<\gamma_{m} \leq n$. The quantum ladder matrix ring associated with $\gamma$, denoted $\mathcal{O}_{q}\left(M_{m, n, \gamma}(\mathbb{k})\right)$, is the $\mathbb{k}$ subalgebra of $\mathcal{O}_{q}\left(M_{m, n}(\mathbb{k})\right)$ generated by the elements $X_{i j} \in \mathcal{O}_{q}\left(M_{m, n}(\mathbb{k})\right)$ such that $(i, j) \in \mathcal{L}_{\gamma}$.

Let us discuss an example to clarify the definition.

EXAMPLE 3.1.3. We put $(m, n)=(3,7)$ and $\gamma=\left\{\gamma_{1}, \gamma_{2}, \gamma_{3}\right\}=\{1,3,6\} \in \Pi_{3,7}$. In the $3 \times 7$ generic matrix $\mathbf{X}=\left(X_{i j}\right)$ associated to $\mathcal{O}_{q}\left(M_{3,7}(\mathbb{k})\right)$, put a bullet on each row as follows: on the first row, the bullet is in column 6 because $\gamma_{3}$ is 6 , on the second row, the bullet is in column 3 because $\gamma_{2}$ is 3 and on the third row, the bullet is in column 1 because $\gamma_{1}=1$. Now, in each position that is to the left of a bullet, or that is below a bullet, put a star. To finish, place $X_{i j}$ in any position $(i, j)$ that has not yet been filled. We obtain

$$
\left(\begin{array}{ccccccc}
* & * & * & * & * & \bullet & X_{17} \\
* & * & \bullet & X_{24} & X_{25} & * & X_{27} \\
\bullet & X_{32} & * & X_{34} & X_{35} & * & X_{37}
\end{array}\right) .
$$

By definition, the ladder quantum matrix ring associated to $\gamma=\{1,3,6\}$ is the subalgebra of $\mathcal{O}_{q}\left(M_{3,7}(\mathbb{k})\right)$ generated by the elements $X_{17}, X_{24}, X_{25}, X_{27}, X_{32}, X_{34}, X_{35}, X_{37}$.

Our aim now is to show that the localisation of $\mathcal{O}_{q}\left(G_{m, n}(\mathbb{k})\right)_{\gamma}$ at the powers of the image of $\gamma$ in $\mathcal{O}_{q}\left(G_{m, n}(\mathbb{k})\right)_{\gamma}$ is isomorphic to a skew Laurent extension of $\mathcal{O}_{q}\left(M_{m, n, \gamma}(\mathbb{k})\right)$.

LEMMA 3.1.4. Let $\gamma=\left\{\gamma_{1}, \ldots, \gamma_{m}\right\} \in \Pi_{m, n}$, with $1 \leq \gamma_{1}<\ldots<\gamma_{m} \leq n$. For $(i, j),(k, l) \in \mathcal{L}_{\gamma}$, the following relations hold in $\mathcal{O}_{q}\left(G_{m, n}(\mathbb{k})\right)$ : 
(i) if $i=k$ and $j<l$, then $m_{i j} m_{k l}=q m_{k l} m_{i j}$;

(ii) if $i<k$ and $j=l$, then $m_{i j} m_{k l}=q m_{k l} m_{i j}$;

(iii) if $i<k$ and $j>l$, then $m_{i j} m_{k l}=m_{k l} m_{i j}$;

(iv) if $i<k$ and $j<l$, then $m_{i j} m_{k l}-m_{k l} m_{i j}=\left(q-q^{-1}\right) m_{i l} m_{k j}$;

(v) $\gamma m_{i j}=q m_{i j} \gamma$.

Proof. The proof is an easy application of Proposition 1.3. We give details for (iv) leaving the other (easier) cases to the reader.

First, note that, if we set $R=\left\{\gamma_{1}, \ldots, \gamma_{m}\right\} \backslash\left\{\gamma_{m+1-i}, \gamma_{m+1-k}\right\}$, then $m_{i j}=$ $\left[\left\{\gamma_{1}, \ldots, \gamma_{m}\right\} \backslash\left\{\gamma_{m+1-i}\right\} \cup\{j\}\right]=\left[R \cup\left\{\gamma_{m+1-k}, j\right\}\right]$ and $m_{k l}=\left[\left\{\gamma_{1}, \ldots, \gamma_{m}\right\} \backslash\left\{\gamma_{m+1-k}\right\} \cup\right.$ $\{l\}]=\left[R \cup\left\{\gamma_{m+1-i}, l\right\}\right]$. In addition, $\gamma_{m+1-i}<j$, since $(i, j)$ and $(k, l)$ are in $\mathcal{L}_{\gamma}$. Hence,

$$
\gamma_{m+1-k}<\gamma_{m+1-i}<j<l \text {. }
$$

It follows that, in $\mathcal{O}_{q}\left(M_{n}(\mathbb{k})\right)$, we have the relation

$$
\begin{aligned}
& {\left[1,2 \mid \gamma_{m+1-k}, j\right]\left[1,2 \mid \gamma_{m+1-i}, l\right]-\left[1,2 \mid \gamma_{m+1-i}, l\right]\left[1,2 \mid \gamma_{m+1-k}, j\right]} \\
& \quad=\left(q-q^{-1}\right)\left[1,2 \mid \gamma_{m+1-k}, l\right]\left[1,2 \mid \gamma_{m+1-i}, j\right] .
\end{aligned}
$$

(This is an immediate consequence of the relation [13][24] - [24][13] $=\left(q-q^{-1}\right)[14][23]$ that holds in $\mathcal{O}_{q}\left(G_{2,4}(\mathbb{k})\right)$, see the introduction of [5], using a suitable injection of $\mathcal{O}_{q}\left(G_{2,4}(\mathbb{k})\right)$ in $\mathcal{O}_{q}\left(M_{n}(\mathbb{k})\right)$.) Now, applying Proposition 1.3 to this relation, with $\bar{P}=$ $\{3, \ldots, m\}$ and $\bar{Q}=R$ gives the relation

$$
\begin{aligned}
{\left[1, \ldots, m \mid R \cup\left\{\gamma_{m+1-k}, j\right\}\right]\left[1, \ldots, m \mid R \cup\left\{\gamma_{m+1-i}, l\right\}\right] } \\
\quad-\left[1, \ldots, m \mid R \cup\left\{\gamma_{m+1-i}, l\right\}\right]\left[1, \ldots, m \mid\left\{R \cup \gamma_{m+1-k}, j\right\}\right] \\
\quad=\left(q-q^{-1}\right)\left[1, \ldots, m \mid R \cup\left\{\gamma_{m+1-k}, l\right\}\right]\left[1, \ldots, m \mid R \cup\left\{\gamma_{m+1-i}, j\right\}\right] .
\end{aligned}
$$

If we view $\mathcal{O}_{q}\left(G_{m, n}(\mathbb{k})\right.$ as the subalgebra of $\mathcal{O}_{q}\left(M_{n}(\mathbb{k})\right)$ generated by the $m \times m$ minors built on the first $m$ rows of the generic matrix of $\mathcal{O}_{q}\left(M_{n}(\mathbb{k})\right)$, this gives the required relation.

REMARK 3.1.5. (i) In view of the defining relations of $\mathcal{O}_{q}\left(M_{m, n}(\mathbb{k})\right)$, it is clear that there exists a $\mathbb{k}$-algebra automorphism $\psi: \mathcal{O}_{q}\left(M_{m, n}(\mathbb{k})\right) \longrightarrow \mathcal{O}_{q}\left(M_{m, n}(\mathbb{k})\right)$ such that $\psi\left(X_{i j}\right)=q X_{i j}$, for each $i, j$.

(ii) Let $\gamma \in \Pi_{m, n}$. It is not difficult to check that the quantum ladder matrix ring, $\mathcal{O}_{q}\left(M_{m, n, \gamma}(\mathbb{k})\right)$, is isomorphic to an iterated skew polynomial extension of $\mathbb{k}$ obtained by inserting the generators $X_{i j}$, with $(i, j) \in \mathcal{L}_{\gamma}$, in lexicographic order. In fact, $\mathcal{O}_{q}\left(M_{m, n, \gamma}(\mathbb{k})\right)$ is isomorphic to the $\mathbb{k}$-algebra generated by indeterminates $X_{i j}$, with $(i, j) \in \mathcal{L}_{\gamma}$, subject to the relations imposed by the fact that $X_{i j} \in \mathcal{O}_{q}\left(M_{m, n}(\mathbb{k})\right)$. In addition $\psi$ clearly restricts to a $\mathbb{k}$-algebra automorphism of $\mathcal{O}_{q}\left(M_{m, n, \gamma}(\mathbb{k})\right)$, that we also denote by $\psi$.

(iii) Let $\gamma \in \Pi_{m, n}$. By point (ii) above and standard results on Gelfand-Kirillov dimension, one has $\operatorname{GKdimO}_{q}\left(M_{m, n, \gamma}(\mathbb{k})\right)=\left|\mathcal{L}_{\gamma}\right|$. Now, clearly, $\psi$ is a locally algebraic automorphism of $\mathcal{O}_{q}\left(M_{m, n, \gamma}(\mathbb{k})\right)$ in the sense of [6, Section 12.3]. It follows, see 
[6, Section 12.3], that:

$\mathrm{GKdim}_{q}\left(M_{m, n, \gamma}(\mathbb{k})\right)\left[Y, Y^{-1} ; \psi\right]=\left|\mathcal{L}_{\gamma}\right|+1=m(n-m)+\frac{m(m+1)}{2}-\left(\sum_{i=1}^{m} \gamma_{i}\right)+1$.

Let $\gamma \in \Pi_{m, n}$. The homomorphic image in $\mathcal{O}_{q}\left(G_{m, n}(\mathbb{k})\right)_{\gamma}$ of an element $x \in$ $\mathcal{O}_{q}\left(G_{m, n}(\mathbb{k})\right)$ will be denoted $\bar{x}$. Theorem 3.1.6 establishes a strong link between the localisation of $\mathcal{O}_{q}\left(G_{m, n}(\mathbb{k})\right)_{\gamma}$ at powers of $\bar{\gamma}$ and a skew Laurent extension of the quantum ladder matrix ring $\mathcal{O}_{q}\left(M_{m, n, \gamma}(\mathbb{k})\right)$. Recall that $\mathcal{O}_{q}\left(G_{m, n}(\mathbb{k})\right)_{\gamma}$ is a quantum graded A.S.L. on the set $\Pi_{m, n} \backslash \Pi_{m, n}^{\gamma}$ (identified with its image in $\left.\mathcal{O}_{q}\left(G_{m, n}(\mathbb{k})\right)_{\gamma}\right)$. The element $\bar{\gamma}$ is the unique minimal element of $\Pi_{m, n} \backslash \Pi_{m, n}^{\gamma}$; and so $\bar{\gamma}$ is a regular normal element of $\mathcal{O}_{q}\left(G_{m, n}(\mathbb{k})\right)_{\gamma}$. Thus, we may form the localisation $\mathcal{O}_{q}\left(G_{m, n}(\mathbb{k})\right)_{\gamma}\left[\bar{\gamma}^{-1}\right]$ of $\mathcal{O}_{q}\left(G_{m, n}(\mathbb{k})\right)_{\gamma}$ at the powers of $\bar{\gamma}$, as indicated in the introduction to Subsection 2.2.

THEOREM 3.1.6. Let $\gamma \in \Pi_{m, n}$. There exists a $\mathbb{k}$-algebra isomorphism

$$
d_{\gamma}: \mathcal{O}_{q}\left(M_{m, n, \gamma}(\mathbb{k})\right)\left[Y, Y^{-1} ; \phi\right] \longrightarrow \mathcal{O}_{q}\left(G_{m, n}(\mathbb{k})\right)_{\gamma}\left[\bar{\gamma}^{-1}\right]
$$

sending $X_{i j}$ to $\overline{m_{i j}}$ and $Y$ to $\bar{\gamma}$.

Proof. The existence of the $\mathbb{k}$-algebra morphism $d_{\gamma}$ is clear from Lemma 3.1.4 and Remark 3.1.5. We now show that $d_{\gamma}$ is onto, by showing that $\mathcal{O}_{q}\left(G_{m, n}(\mathbb{k})\right)_{\gamma}\left[\bar{\gamma}^{-1}\right]$ is generated as a $\mathbb{k}$-algebra by $\bar{\gamma}, \bar{\gamma}^{-1}$ and $\overline{m_{i j}},(i, j) \in \mathcal{L}_{\gamma}$. Clearly, $\mathcal{O}_{q}\left(G_{m, n}(\mathbb{k})\right)_{\gamma}\left[\bar{\gamma}^{-1}\right]$ is generated as a $\mathbb{k}$-algebra by $\bar{\gamma}, \bar{\gamma}^{-1}$ and the images in $\mathcal{O}_{q}\left(G_{m, n}(\mathbb{k})\right)_{\gamma}$ of the minors in $\Pi_{m, n} \backslash \Pi_{m, n}^{\gamma}$; so, what we must show is that $\bar{x} \in \operatorname{im} d_{\gamma}$, for all $x \in \Pi_{m, n} \backslash \Pi_{m, n}^{\gamma}$. To each $x=\left[j_{1}, \ldots, j_{m}\right] \in \Pi_{m, n} \backslash \Pi_{m, n}^{\gamma}$, we associate the number $n(x)$ of elements $j_{s}$, with $1 \leq s \leq$ $m$, such that $j_{s} \notin\left\{\gamma_{1}, \ldots, \gamma_{m}\right\}$. As mentioned in Remark 3.1.1, if $x \in \Pi_{m, n} \backslash \Pi_{m, n}^{\gamma}$ is such that $n(x) \leq 1$, then either $\bar{x}=\bar{\gamma}$ or $\bar{x}=\overline{m_{i j}}$ for some $(i, j) \in \mathcal{L}_{\gamma}$; so that $\bar{x} \in \operatorname{im} d_{\gamma}$. Now, suppose $t$ is an integer in $\{1, \ldots, m-1\}$ with the property that any $x \in \Pi_{m, n} \backslash \Pi_{m, n}^{\gamma}$ such that $n(x) \leq t$ satisfies $\bar{x} \in \operatorname{im} d_{\gamma}$. Consider $x=\left[j_{1}, \ldots, j_{m}\right] \in \Pi_{m, n} \backslash \Pi_{m, n}^{\gamma}$ such that $n(x)=t+1$. In addition, let $1 \leq \ell \leq m$ be such that $j_{\ell} \notin\left\{\gamma_{1}, \ldots, \gamma_{m}\right\}$. The generalised quantum Plücker relations of [5, Theorem 2.1], applied with $J_{1}=\emptyset, J_{2}=\left\{j_{1}, \ldots, j_{m}\right\} \backslash$ $\left\{j_{\ell}\right\}$ and $K=\left\{\gamma_{1}, \ldots, \gamma_{m}\right\} \cup\left\{j_{\ell}\right\}$ give a relation

$$
\sum_{K^{\prime} \sqcup K^{\prime \prime}=K}(-q)^{\bullet}\left[K^{\prime}\right]\left[K^{\prime \prime} \sqcup J_{2}\right]=0
$$

in $\mathcal{O}_{q}\left(G_{m, n}(\mathbb{k})\right)$. (Here, by a symbol $(-q)^{\bullet}$, we mean some power of $-q$ with exponent in $\mathbb{Z}$.) Let us now consider the various terms $\left[K^{\prime}\right]\left[K^{\prime \prime} \sqcup J_{2}\right]$ of the above equation. When $K^{\prime \prime}=\left\{j_{\ell}\right\}$, then $\left[K^{\prime}\right]\left[K^{\prime \prime} \sqcup J_{2}\right]=\gamma x$. Otherwise, $K^{\prime \prime}=\left\{\gamma_{k}\right\}$ for some $1 \leq k \leq m$ such that $\gamma_{k} \notin\left\{j_{1}, \ldots, j_{m}\right\}$. In this case, $\left[K^{\prime}\right]\left[K^{\prime \prime} \sqcup J_{2}\right]=\left[\left\{\gamma_{1}, \ldots, \gamma_{m}\right\} \backslash\left\{\gamma_{k}\right\} \cup\left\{j_{\ell}\right\}\right]\left[\left\{j_{1}, \ldots, j_{m}\right\} \backslash\right.$ $\left.\left\{j_{\ell}\right\} \cup\left\{\gamma_{k}\right\}\right]$ (notice that the image of such a term in $\mathcal{O}_{q}\left(G_{m, n}(\mathbb{k})\right)_{\gamma}$ might very well be zero). Hence, taking the image of the above relation in $\mathcal{O}_{q}\left(G_{m, n}(\mathbb{k})\right)_{\gamma}$, we get a relation of the form

$$
\overline{\gamma \bar{x}}=\sum(-q)^{\bullet} \overline{y z}
$$

where the sum extends over pairs $(y, z)$ of elements in $\Pi_{m, n} \backslash \Pi_{m, n}^{\gamma}$ such that $n(y)=1$ and $n(z)=t$. Hence, each $\bar{x} \in \operatorname{im}\left(d_{\gamma}\right)$, by the induction hypothesis since $\bar{\gamma}$ is invertible in $\mathcal{O}_{q}\left(G_{m, n}(\mathbb{k})\right)_{\gamma}\left[\bar{\gamma}^{-1}\right]$. This shows that $d_{\gamma}$ is surjective. Recall from Remark 3.1.5 that $\operatorname{GKdim} \mathcal{O}_{q}\left(M_{m, n, \gamma}(\mathbb{k})\right)\left[Y, Y^{-1} ; \psi\right]=m(n-m)+\frac{m(m+1)}{2}-\left(\sum_{i=1}^{m} \gamma_{i}\right)+1$. On the 
other hand, $\bar{\gamma}$ is a local normal element in the sense of [6, Section 12.4]. Hence, by [6, Theorem 12.4.4] and Corollary 2.1.6, $\operatorname{GKdim}_{q}\left(G_{m, n}(\mathbb{k})\right)_{\gamma}\left[\bar{\gamma}^{-1}\right]=m(n-m)+$ $\frac{m(m+1)}{2}-\left(\sum_{i=1}^{m} \gamma_{i}\right)+1$. As $\mathcal{O}_{q}\left(M_{m, n, \gamma}(\mathbb{k})\right)\left[Y, Y^{-1} ; \psi\right]$ is an integral domain and $d_{\gamma}$ is surjective, the injectivity of $d_{\gamma}$ follows, by [6, Proposition 3.15].

COROLlary 3.1.7. Let $\gamma \in \Pi_{m, n}$. The $\mathbb{k}$-algebra $\mathcal{O}_{q}\left(G_{m, n}(\mathbb{k})\right)_{\gamma}$ is a normal domain.

Proof. Consider $\tau \in \Pi_{m, n}$. Theorem 3.1.6 asserts that there exists a $\mathbb{k}$-algebra isomorphism $\mathcal{O}_{q}\left(M_{m, n, \tau}(\mathbb{k})\right)\left[Y, Y^{-1} ; \psi\right] \cong \mathcal{O}_{q}\left(G_{m, n}(\mathbb{k})\right)_{\tau}\left[\bar{\tau}^{-1}\right]$. Hence $\mathcal{O}_{q}\left(G_{m, n}(\mathbb{k})\right)_{\tau}\left[\bar{\tau}^{-1}\right]$ is an integral domain. As we already mentioned, $\bar{\tau}$ is a regular element of $\mathcal{O}_{q}\left(G_{m, n}(\mathbb{k})\right)_{\tau}$; so the canonical map $\mathcal{O}_{q}\left(G_{m, n}(\mathbb{k})\right)_{\tau} \longrightarrow \mathcal{O}_{q}\left(G_{m, n}(\mathbb{k})\right)_{\tau}\left[\bar{\tau}^{-1}\right]$ is an injection. It follows that $\mathcal{O}_{q}\left(G_{m, n}(\mathbb{k})\right)_{\tau}$ is an integral domain. Hence, we have proved that any quantum Schubert variety is an integral domain.

It follows that, in the notation of Subsection 2.2, the ideal $I_{\tau}$ of $\mathcal{O}_{q}\left(G_{m, n}(\mathbb{k})\right)_{\gamma}$ is a completely prime ideal for all $\tau \in \Pi_{m, n} \backslash \Pi_{m, n}^{\gamma}$. Moreover, since $\mathcal{O}_{q}\left(G_{m, n}(\mathbb{k})\right)_{\gamma}\left[\bar{\gamma}^{-1}\right]$ is isomorphic to a localisation of an iterated Ore extension of $\mathbb{k}$, it is a normal domain by [12, V. Proposition 2.5, IV Proposition 2.1]. Hence, Proposition 2.2.2 applies to the quantum graded A.S.L. $\mathcal{O}_{q}\left(G_{m, n}(\mathbb{k})\right)_{\gamma}$ (whose underlying poset has a single minimal element, as noticed in Remark 2.1.4); so we conclude that $\mathcal{O}_{q}\left(G_{m, n}(\mathbb{k})\right)_{\gamma}$ is a normal domain.

3.2. The AS-Cohen-Macaulay and AS-Gorenstein properties. The following result is Theorem 4.2 of [10].

THEOREM 3.2.1. Let $\gamma \in \Pi_{m, n}$. The quantum Schubert variety $\mathcal{O}_{q}\left(G_{m, n}(\mathbb{k})\right)_{\gamma}$ is $A S$ Cohen-Macaulay.

It is now easy to determine among quantum Schubert varieties those that are AS-Gorenstein. Let $A$ be a noetherian $\mathbb{N}$-graded connected $\mathbb{k}$-algebra. For the definition of the AS-Gorenstein condition for $A$ see [10, Subsection 2.1]. Suppose in addition that $A$ has enough normal elements in the sense of Zhang, see [10, Definition 2.1.3]. Then, $A$ is AS-Gorenstein if and only if it has finite injective dimension on both sides. In particular, if $A$ is commutative, then $A$ is AS-Gorenstein if and only if it is Gorenstein in the usual sense. For details on these statements, see Subsection 2.1 of [10] and in particular [10, Remark 2.1.10].

We need to introduce some more notation. Let $\gamma=\left\{\gamma_{1}, \ldots, \gamma_{m}\right\} \in \Pi_{m, n}$. Following $\left[1\right.$, Chapter 6 , Section B], we denote by $\beta_{0}, \ldots, \beta_{s}$ the blocks of consecutive integers in $\gamma$ and by $\chi_{0}, \ldots, \chi_{s-1}$ the gaps between these blocks.

THEOREM 3.2.2. Let $\gamma=\left\{\gamma_{1}, \ldots, \gamma_{m}\right\} \in \Pi_{m, n}$. In the previous notation, we put $t=s$ if $\gamma_{m}<n$ and $t=s-1$ if $\gamma_{m}=n$. Then, the $\mathbb{k}$-algebra $\mathcal{O}_{q}\left(G_{m, n}(\mathbb{k})\right)_{\gamma}$ is AS-Gorenstein if and only if (with the above notation) $\left|\chi_{i-1}\right|=\left|\beta_{i}\right|$ for $1 \leq i \leq t$.

Proof. As mentioned above, $\mathcal{O}_{q}\left(G_{m, n}(\mathbb{k})\right)_{\gamma}$ is a quantum graded algebra with a straightening law on the poset $\Pi_{m, n} \backslash \Pi_{m, n}^{\gamma}$. It follows from [10, Remark 2.1.4] that it has enough normal elements. In addition, $\mathcal{O}_{q}\left(G_{m, n}(\mathbb{k})\right)_{\gamma}$ is a AS-Cohen-Macaulay domain, by Corollary 3.1.7 and Theorem 3.2.1. On the other hand, $\mathcal{O}_{q}\left(G_{m, n}(\mathbb{k})\right)_{\gamma}$ has a vector space basis consisting of standard monomials on $\Pi_{m, n} \backslash \Pi_{m, n}^{\gamma}$, since it is a quantum graded algebra with a straightening law on this poset. Clearly, this implies that the Hilbert series of $\mathcal{O}_{q}\left(G_{m, n}(\mathbb{k})\right)_{\gamma}$ is independent of the particular value 
of $q \in \mathbb{k}^{*}$. Hence, using [2, Theorem 6.2], $\mathcal{O}_{q}\left(G_{m, n}(\mathbb{k})\right)_{\gamma}$ is AS-Gorenstein if and only if $\mathcal{O}_{1}\left(G_{m, n}(\mathbb{k})\right)_{\gamma}$ is AS-Gorenstein; that is, if and only if $\mathcal{O}_{1}\left(G_{m, n}(\mathbb{k})\right)_{\gamma}$ is Gorenstein (by the discussion above). The result now follows from Corollary 8.13 of [1] .

4. Application to the normality of quantum determinantal rings. The aim of this section is to apply the previous results on quantum Schubert varieties in order to show that quantum determinantal rings are normal domains. Recall that a quantum determinantal ring is a factor $\mathcal{O}_{q}\left(M_{m, n}(\mathbb{k})\right) / \mathcal{I}_{t}$, where $1 \leq t \leq m$ and $\mathcal{I}_{t}$ is the ideal of $\mathcal{O}_{q}\left(M_{m, n}(\mathbb{k})\right)$ generated by the $t \times t$ quantum minors.

Let us start by defining a larger class of quotients of $\mathcal{O}_{q}\left(M_{m, n}(\mathbb{k})\right)$. This class is obtained from $\mathcal{O}_{q}\left(M_{m, n}(\mathbb{k})\right)$ in the same way as quantum Schubert varieties are obtained from $\mathcal{O}_{q}\left(G_{m, n}(\mathbb{k})\right)$.

DeFINITION 4.1. Let $\delta \in \Delta_{m, n}$ and put $\Delta_{m, n}^{\delta}=\left\{\alpha \in \Delta_{m, n} \mid \alpha \Varangle_{\text {st }} \delta\right\}$.

$$
\mathcal{O}_{q}\left(M_{m, n}(\mathbb{k})\right)_{\delta}=\mathcal{O}_{q}\left(M_{m, n}(\mathbb{k})\right) /\left\langle\Delta_{m, n}^{\delta}\right\rangle .
$$

REMARK 4.2. (i) Let $\delta \in \Delta_{m, n}$. It is clear that $\Delta_{m, n}^{\delta}$ is a $\Delta_{m, n}$-ideal. Hence, as mentioned in Subsection 2.1, the generalised quantum determinantal ring $\mathcal{O}_{q}\left(M_{m, n}(\mathbb{k})\right)_{\delta}$ inherits from $\mathcal{O}_{q}\left(M_{m, n}(\mathbb{k})\right)$ the structure of a quantum graded A.S.L. on the poset $\Delta_{m, n} \backslash \Delta_{m, n}^{\delta}$. Notice also that $\Delta_{m, n} \backslash \Delta_{m, n}^{\delta}$ has a unique minimal element.

(ii) Let $\delta \in \Delta_{m, n}$. It follows from point (i) above that $\mathcal{O}_{q}\left(M_{m, n}(\mathbb{k})\right)_{\delta}$ has a standard monomial basis inherited from the corresponding basis of $\mathcal{O}_{q}\left(M_{m, n}(\mathbb{k})\right)$. The elements of this $\mathbb{k}$-basis are the canonical images in $\mathcal{O}_{q}\left(M_{m, n}(\mathbb{k})\right)_{\delta}$ of the standard monomials of $\mathcal{O}_{q}\left(M_{m, n}(\mathbb{k})\right)$ that either are equal to 1 or are of the form $\left[I_{1} \mid J_{1}\right] \ldots\left[I_{t} \mid J_{t}\right]$ with $\delta \leq_{\text {st }}\left[I_{1} \mid J_{1}\right]$. Then,

(iii) Let $\delta=\left(\left\{i_{1}<\ldots<i_{r}\right\},\left\{j_{1}<\ldots<j_{r}\right\}\right)$, for some integer $r$ such that $1 \leq r \leq m$.

$$
\mathrm{GKdim}_{q}\left(M_{m, n}(\mathbb{k})\right)_{\delta}=(m+n) r-\sum_{s=1}^{r}\left(i_{s}+j_{s}\right)+r,
$$

by Proposition 2.1.5 and [1, 5.12].

(iv) As noticed in [10, Section 3.5], quantum determinantal rings are special cases of generalised quantum determinantal rings, hence justifying the vocabulary in Definition 4.1. More precisely, the paragraph before [10, Corollary 3.5.4] shows that, for $1<t \leq$ $m$, the quantum determinantal $\operatorname{ring} \mathcal{O}_{q}\left(M_{m, n}(\mathbb{k})\right) / \mathcal{I}_{t}$ is equal to the generalised quantum determinantal $\operatorname{ring} \mathcal{O}_{q}\left(M_{m, n}(\mathbb{k})\right)_{\delta}$ where $\delta=(\{1, \ldots, t-1\},\{1, \ldots, t-1\})$.

The normality of quantum determinantal rings will be establised by applying Proposition 2.2.2. Hence, we first need to prove that generalised quantum determinantal rings are integral domains. This is what we do now. Fix an element $\delta \in \Delta_{m, n}$ and denote by $\gamma$ the image of $\delta$ under the map $\delta_{m, n}$ introduced in Section 1. Recall from Section 1 the dehomogenisation map

$$
D_{m, n}: \mathcal{O}_{q}\left(M_{m, n}(\mathbb{k})\right)\left[y, y^{-1} ; \phi\right] \longrightarrow \mathcal{O}_{q}\left(G_{m, m+n}(\mathbb{k})\right)\left[[M]^{-1}\right]
$$

that sends $[I \mid J]$ to $\left[K_{(I, J)}\right][M]^{-1}$ and $y$ to $[M]$. It is clear that the ideal $\left\langle\Delta_{m, n}^{\delta}\right\rangle$ of $\mathcal{O}_{q}\left(M_{m, n}(\mathbb{k})\right)$ is stable under $\phi$. Hence, using the obvious abuse of notation, there is a 
canonical isomorphism

$$
\begin{aligned}
\mathcal{O}_{q}\left(M_{m, n}(\mathbb{k})\right)\left[y, y^{-1} ; \phi\right] /\left\langle\Delta_{m, n}^{\delta}\right\rangle\left[y, y^{-1} ; \phi\right] & \cong\left(\mathcal{O}_{q}\left(M_{m, n}(\mathbb{k})\right) /\left\langle\Delta_{m, n}^{\delta}\right\rangle\right)\left[y, y^{-1} ; \phi\right] \\
& =\mathcal{O}_{q}\left(M_{m, n}(\mathbb{k})\right)_{\delta}\left[y, y^{-1} ; \phi\right] .
\end{aligned}
$$

(Here, the notation $\left\langle\Delta_{m, n}^{\delta}\right\rangle\left[y, y^{-1} ; \phi\right]$ on the left hand side stands for the two-sided ideal of $\mathcal{O}_{q}\left(M_{m, n}(\mathbb{k})\right)\left[y, y^{-1} ; \phi\right]$ generated by $\Delta_{m, n}^{\delta}$. Now, observe that the ideal $\left\langle\Pi_{m, n+m}^{\gamma}\right\rangle$ of $\mathcal{O}_{q}\left(G_{m, m+n}(\mathbb{k})\right)$ is completely prime, by Corollary 3.1.7, and does not intersect the set of powers of $[M]$, as one may easily prove using the standard basis of $\mathcal{O}_{q}\left(G_{m, m+n}(\mathbb{k})\right)$. Hence, there is a canonical isomorphism

$$
\begin{aligned}
\mathcal{O}_{q}\left(G_{m, m+n}(\mathbb{k})\right)\left[[M]^{-1}\right] /\left\langle\Pi_{m, n}^{\gamma}\right\rangle\left[[M]^{-1}\right] & \cong\left(\mathcal{O}_{q}\left(G_{m, m+n}(\mathbb{k})\right) /\left\langle\Pi_{m, n}^{\gamma}\right\rangle\right)\left[\overline{[M]}^{-1}\right] \\
& =\mathcal{O}_{q}\left(G_{m, m+n}(\mathbb{k})\right)_{\gamma}\left[\left[\overline{[M]}{ }^{-1}\right] .\right.
\end{aligned}
$$

(Recall that, if $x \in \mathcal{O}_{q}\left(G_{m, m+n}(\mathbb{k})\right)$, then $\bar{x}$ stands for its canonical image in $\mathcal{O}_{q}\left(G_{m, m+n}(\mathbb{k})\right)_{\gamma}$. The same convention applies to $\left.\mathcal{O}_{q}\left(M_{m, n}(\mathbb{k})\right)_{\delta}.\right)$

Clearly, $\left.D_{m, n}\left(\left\langle\Delta_{m, n}^{\delta}\right\rangle\left[y, y^{-1} ; \phi\right]\right)=\left\langle\Pi_{m, n}^{\gamma}\right\rangle\right)\left[[M]^{-1}\right]$; and so it follows that $D_{m, n}$ induces an isomorphism

$$
D_{m, n}^{\delta}: \mathcal{O}_{q}\left(M_{m, n}(\mathbb{k})\right)_{\delta}\left[y, y^{-1} ; \phi\right] \longrightarrow \mathcal{O}_{q}\left(G_{m, m+n}(\mathbb{k})\right)_{\gamma}\left[\overline{[M]}^{-1}\right]
$$

that sends $\overline{[I \mid J]}$ to ${\overline{\left[K_{(I, J)}\right]}}_{[M]}^{-1}$ and $y$ to $\overline{[M]}$.

From this we deduce the following result.

Proposition 4.3. Let $\delta \in \Delta_{m, n}$. Then the ring $\mathcal{O}_{q}\left(M_{m, n}(\mathbb{k})\right)_{\delta}$ is an integral domain.

Proof. Let $\gamma$ be the image of $\delta$ under the map $\delta_{m, n}$. By the above discussion, we have an isomorphism

$$
D_{m, n}^{\delta}: \mathcal{O}_{q}\left(M_{m, n}(\mathbb{k})\right)_{\delta}\left[y, y^{-1} ; \phi\right] \longrightarrow \mathcal{O}_{q}\left(G_{m, m+n}(\mathbb{k})\right)_{\gamma}\left[\overline{[M]}^{-1}\right] .
$$

Hence, the result follows by Corollary 3.1.7.

The normality of quantum determinantal rings is now easy to obtain. First, we need a lemma.

Lemma 4.4. Let $2 \leq t \leq m$. Let $\delta=[\{1, \ldots, t-1\} \mid\{1, \ldots, t-1\}] \in \mathcal{O}_{q}\left(M_{m, n}(\mathbb{k})\right)$ and let $\bar{\delta}$ be the canonical image of $\delta$ in $\mathcal{O}_{q}\left(M_{m, n}(\mathbb{k})\right) / \mathcal{I}_{t}$. Let $A_{t}$ be the subalgebra of $\mathcal{O}_{q}\left(M_{m, n}(\mathbb{k})\right)$ generated by the elements $X_{i j}$ such that either $i \leq t-1$ or $j \leq t-1$. Then $\delta$ is a normal element in $A_{t}$ and

$$
\left(\mathcal{O}_{q}\left(M_{m, n}(\mathbb{k})\right) / \mathcal{I}_{t}\right)\left[\bar{\delta}^{-1}\right] \cong A_{t}\left[\delta^{-1}\right] .
$$

Proof. For $2 \leq t \leq m$, there is an obvious algebra homomorphism

$$
A_{t} \longrightarrow \mathcal{O}_{q}\left(M_{m, n}(\mathbb{k})\right) \longrightarrow \mathcal{O}_{q}\left(M_{m, n}(\mathbb{k})\right) / \mathcal{I}_{t} \longrightarrow\left(\mathcal{O}_{q}\left(M_{m, n}(\mathbb{k})\right) / \mathcal{I}_{t}\right)\left[\bar{\delta}^{-1}\right]
$$

Here, the first map is the obvious injection, the second is the canonical projection while the third is the natural injection of a ring into a localisation with respect to the multiplicative set generated by a regular normal element. In addition, $\delta$ is a normal 
element of $A_{t}$, see [13, Lemma 4.5.1], and it is sent to an invertible element by the above map. Hence, there is a natural algebra homomorphism

$$
\phi_{t}: A_{t}\left[\delta^{-1}\right] \longrightarrow\left(\mathcal{O}_{q}\left(M_{m, n}(\mathbb{k})\right) / \mathcal{I}_{t}\right)\left[\bar{\delta}^{-1}\right]
$$

We will show that $\phi_{t}$ is an isomorphism. First, we show that $\phi_{t}$ is surjective. Clearly, $\bar{\delta}^{-1}$ is in the image of $\phi_{t}$. Let $1 \leq i \leq m$ and $1 \leq j \leq n$. It is clear that if either $i<t$ or $j<t$, then $X_{i j}+\mathcal{I}_{t}$ is in the image of $\phi_{t}$. Now, assume $i, j \geq t$. The subalgebra of $\mathcal{O}_{q}\left(M_{m, n}(\mathbb{k})\right)$ generated by the entries of the generic matrix corresponding to rows $1, \ldots, t-1, i$ and columns $1, \ldots, t-1, j$ is isomorphic to $\mathcal{O}_{q}\left(M_{t}(\mathbb{k})\right)$. We may then develop its quantum determinant with respect to its last row. This relation, seen inside $\left(\mathcal{O}_{q}\left(M_{m, n}(\mathbb{k})\right) / \mathcal{I}_{t}\right)\left[\bar{\delta}^{-1}\right]$, shows that $X_{i j}+\mathcal{I}_{t}$ is in the image of $\phi_{t}$.

Next we show that $\phi_{t}$ is injective. First, it is easy to see that

$$
\mathrm{GKdim} A_{t}=m n-(m-(t-1))(n-(t-1)) .
$$

In addition, since $\delta$ commutes up to scalar with each of the canonical generators of $A_{t}$, it is easy to show that it is a local normal element in the sense of [6, Section 12.4]. It then follows by [6, Theorem 12.4.4.] that $\operatorname{GKdim}\left(A_{t}\right)_{\delta}=m n-(m-(t-1))(n-$ $(t-1))$. On the other hand, by Remark 4.2, $\operatorname{GKdim} \mathcal{O}_{q}\left(M_{m, n}(\mathbb{k})\right) / \mathcal{I}_{t}=m n-(m-(t-$ $1))(n-(t-1))$ from which it follows that $\operatorname{GKdim}\left(\mathcal{O}_{q}\left(M_{m, n}(\mathbb{k})\right) / \mathcal{I}_{t}\right)\left[\bar{\delta}^{-1}\right] \geq m n-(m-$ $(t-1))(n-(t-1))$, see [6, Lemma 3.1]. As $A_{t}\left[\delta^{-1}\right]$ is an integral domain, any non-zero element of $A_{t}\left[\delta^{-1}\right]$ is regular. Hence, in view of the above estimates of Gelfand-Kirillov dimension, by [6, Proposition 3.15] we must have $\operatorname{ker} \phi_{t}=(0)$.

THEOREM 4.5. Let $1 \leq t \leq m$. Then $\mathcal{O}_{q}\left(M_{m, n}(\mathbb{k})\right) / \mathcal{I}_{t}$ is a normal domain.

Proof. The case $t=1$ is trivial. Let $2 \leq t \leq m$. Recall that $\mathcal{O}_{q}\left(M_{m, n}(\mathbb{k})\right) / \mathcal{I}_{t}=$ $\mathcal{O}_{q}\left(M_{m, n}(\mathbb{k})\right)_{\delta}$, where $\delta=(\{1, \ldots, t-1\},\{1, \ldots, t-1\})$. Hence, $\mathcal{O}_{q}\left(M_{m, n}(\mathbb{k})\right) / \mathcal{I}_{t}$ is a domain by Proposition 4.3. In addition, we are in the context of Section 2.2 since $\mathcal{O}_{q}\left(M_{m, n}(\mathbb{k})\right) / \mathcal{I}_{t}$ is a quantum graded A.S.L. whose underlying poset has a single minimal element, namely $\bar{\delta}=\delta+\mathcal{I}_{t}$. In addition, Proposition 4.3 shows that, for any upper neighbour $\gamma$ of $\bar{\delta}$, the $\operatorname{ring}\left(\mathcal{O}_{q}\left(M_{m, n}(\mathbb{k})\right) / \mathcal{I}_{t}\right) / I_{\gamma}$ is a domain (here, we are using the notation of Subsection 2.2). By Proposition 2.2.2, it is enough to show that $\left(\mathcal{O}_{q}\left(M_{m, n}(\mathbb{k})\right) / \mathcal{I}_{t}\right)\left[\bar{\delta}^{-1}\right]$ is a normal domain. However, using [12, V. Proposition 2.5, IV. Proposition 2.1], this is a consequence of Lemma 4.4.

REMARK 4.6. In the light of Theorem 4.5, one obvious question arises: are generalised quantum determinantal rings normal domains? Recall that this is true in the commutative case, see [1, Theorem 6.3]. Let $\delta \in \Delta_{m, n}$. The isomorphism $D_{m, n}^{\delta}$ together with Corollary 3.1.7 shows that the skew Laurent extension $\mathcal{O}_{q}\left(M_{m, n}(\mathbb{k})\right)_{\delta}\left[y, y^{-1} ; \phi\right]$ is normal. However, we have not been able to deduce that $\mathcal{O}_{q}\left(M_{m, n}(\mathbb{k})\right)_{\delta}$ is normal from this fact. Another approach to the normality of $\mathcal{O}_{q}\left(M_{m, n}(\mathbb{k})\right)_{\delta}$ (that is the approach we have used to derive the normality of quantum determinantal rings) would be to apply Proposition 2.2.2. We would then need to show that $\mathcal{O}_{q}\left(M_{m, n}(\mathbb{k})\right)_{\delta}\left[\bar{\delta}^{-1}\right]$ is a normal domain. The problem here is of technical nature: presumably, $\mathcal{O}_{q}\left(M_{m, n}(\mathbb{k})\right)_{\delta}\left[\bar{\delta}^{-1}\right]$ can be described as a localisation of an iterated Ore extension (this would generalise Lemma 4.4 to any generalised quantum determinantal ring). However, getting such a description seems to be a rather tricky computation. 


\section{REFERENCES}

1. W. Bruns and U. Vetter, Determinantal rings, Lecture Notes in Mathematics, No. 1327 (Springer-Verlag, 1988).

2. P. Jørgensen and J.J. Zhang, Gourmet's guide to Gorensteinness, Adv. in Math. 151 (2000), no. 2, 313-345.

3. N. Gonciulea and V. Lakshmibai, Flag Varieties, Actualités Mathématiques (Hermann, Paris, 2001).

4. R. Q. Huang and J. J. Zhang, Standard basis theorem for quantum linear groups, $A d v$. in Math. 102 (1993), 202-229.

5. A. Kelly, T.H. Lenagan and L. Rigal. Ring theoretic properties of quantum grassmannians. J. Algebra Appl. 3 (2004), 9-30.

6. G. Krause and T.H. Lenagan, Growth of algebras and Gelfand-Kirillov dimension. Revised edition. Graduate Studies in Mathematics, 22 (American Mathematical Society, Providence, RI, 2000)

7. D. Krob and B. Leclerc, Minor identities for quasi-determinants and quantum determinants. Comm. Math. Phys. 169 (1995), 1-23.

8. V. Lakshmibai and N. Reshetikhin. Quantum deformations of $S L_{n} / B$ and its Schubert varieties, Special functions (Okayama, 1990), 149-168, ICM-90 Satell. Conf. Proc. (SpringerVerlag, 1991).

9. T.H. Lenagan and L. Rigal, The maximal order property for quantum determinantal rings, Proc. Edinburgh Math. Soc. (2) 46 (2003), 513-529.

10. T.H. Lenagan and L. Rigal, Quantum graded algebras with a straightening law and the AS-Cohen-Macaulay property for quantum determinantal rings and quantum grassmannians, J. Algebra 301 (2006), 670-702.

11. J.C. McConnell and J.C. Robson, Noncommutative Noetherian rings, Revised edition. Graduate Studies in Mathematics, vol. 30 (American Mathematical Society, Providence, RI, 2001).

12. G. Maury and J. Raynaud, Ordres maximaux au sens de K. Asano, Lecture Notes in Mathematics, No. 808 (Springer-Verlag, 1980). 439.

13. B. Parshall and J. Wang, Quantum linear groups, Mem. Amer. Math. Soc. 89 (1991), no.

14. L. Rigal, Normalité de certains anneaux déterminantiels quantiques, Proc. Edinburgh Math. Soc. (2) 42 (1999), 621-640. 